\title{
Development of a Traceable Cantilever Calibration Device
}

\author{
Oliver Dannberg', Michael Kühnel ${ }^{2}$, Thomas Fröhlich ${ }^{1}$ \\ ${ }^{1}$ Technische Universität IImenau, IImenau, Germany, \\ ${ }^{2}$ SIOS Meßtechnik GmbH, IImenau, Germany \\ oliver.dannberg@tu-ilmenau.de
}

\begin{abstract}
Summary:
This paper describes the development of a device that measures the force-displacement-curve of cantilevers to determine their stiffness. An interferometer detects the deflection of the cantilever while the force is measured by a novel EMK load cell with a soft mechanism to provide a high force resolution. The results of first cantilever calibrations with this device showed a low uncertainty and no detectable damage to the tip.
\end{abstract}

Keywords: Cantilever calibration, load cell, force measurements, interferometer

\section{Introduction}

The measurement of forces in the subnanoand nanonewton range is a strongly growing application in different fields of science such as biology, biophysics, medical- or materials science. Typically, these forces are determined by measuring the deflection of calibrated AFM cantilevers. However, an uncertainty of the cantilever stiffness has a direct impact to the force measurement.

This paper describes the development of a novel device that is able to do a traceable cantilever calibration with small uncertainties.

\section{Cantilever calibration}
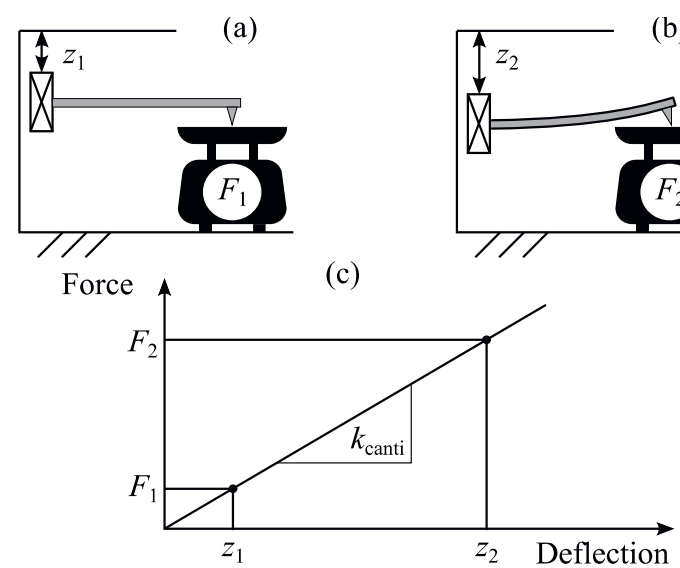

Fig. 1: Static experimental calibration setup for two load steps $(a, b)$ with the corresponding force-deflection-curve (c).

The most accurate way to calibrate the stiffness of cantilevers is a static experimental calibration process [1] as shown in Figure 1. The cantilever is attached to a piezo stage and moved in z-direction while the tip is touching an electromagnetic force compensating (EMFC) balance. Since the weighting pan of the balance is always controlled to the same position the movement of the cantilever equals its deflection [2]. The slope of the force-deflection-curve represents the stiffness of the cantilever.

For the calibration process, the cantilever is clamped to a holder that is positioned in $\mathrm{x}, \mathrm{y}$ and $z$ while an interferometer measures its $z-$ position. High calibration forces cause a nonlinear force-deflection-curve and wear of the cantilever tip [5]. Therefore, a high resolution load cell has been developed that achieves a small uncertainty, even for small calibration forces.

\section{Force Measurement}

The cantilever force is measured by a custom load cell that is optimized to have a high force resolution. Therefore it uses two major elements that are different, compared to commercial load cells:

1. a single joint mechanism with a low stiffness of $1 \mathrm{Nm}^{-1}$ at the load button

2. a difference interferometer (SIOS SP $2000 \mathrm{DI})$ to measure the beam deflection with a resolution of $10 \mathrm{pm}$

Two mirrors (8) on top and bottom of the load cell (4) reflect the interferometer beams. Figure 2 shows the CAD model of the custom load cell. A deflection of the load cell increases the length of one beam while the other one decreases. 
The symmetry of the optical path reduces the sensitivity for external disturbances such as changes in temperature, humidity and air pressure. An additional positioning sensor measures the absolute zero position (1) with an accuracy of $1 \mathrm{~nm}$ [3].

The center of gravity is adjusted in horizontal and vertical direction by means of two adjusting screws (3), (5). This makes the balance less sensitive to tilt and other disturbances.

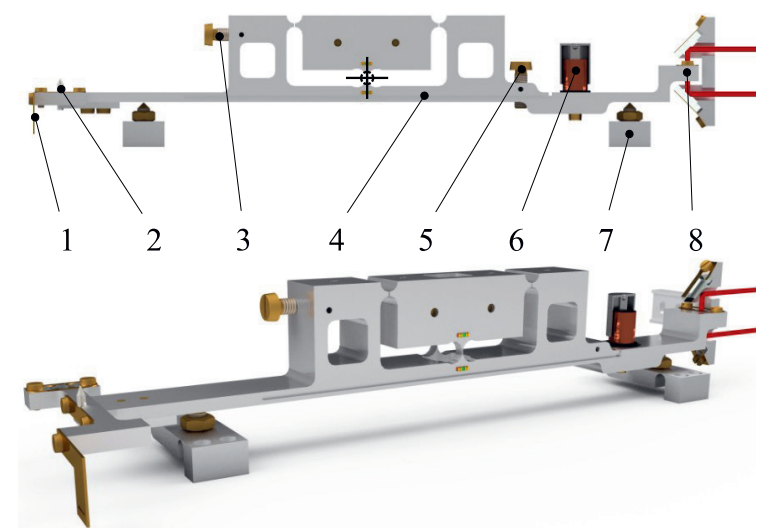

Fig. 2: $C A D$ model of the single pivot beam balance: (1) Absolute position sensor; (2) Diamond load button; (3) Horizontal adjustment weight; (4) Monolithic beam; (5) Vertical adjustment weight; (6) Voice coil; (7) Mechanical limits; (8) Mirrors.

A voice coil (6) with a force constant of $26 \mathrm{mNA}^{-1}$ generates a Lorenz force to compensate the cantilever force that is applied to a diamond load button (2) with the shape of a truncated cone. The tip touches the circular, flat surface with a diameter of $10 \mu \mathrm{m}$ which ensures a constant leverage ratio.

\section{Position measurement}

For the cantilever calibration, the cantilever is clamped to a cantilever holder.

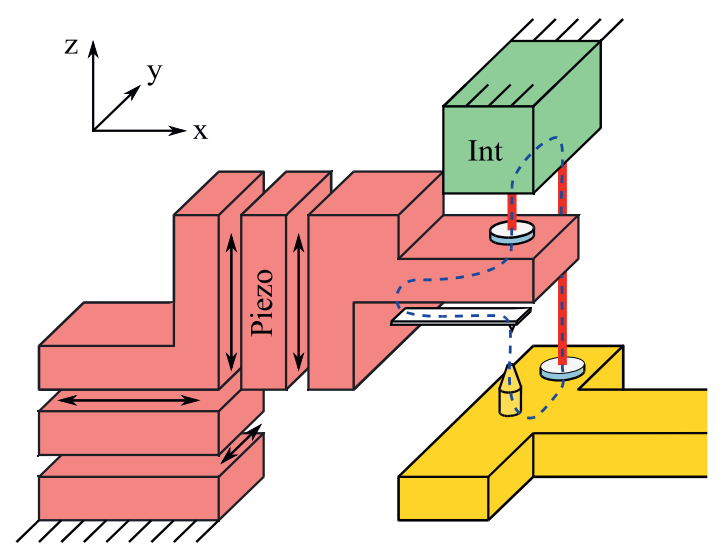

Fig. 3: Positioning of the cantilever and the measurement of its z-position with the measurement loop (blue)
A stack of three stages moves the cantilever in $x, y$ and $z$ direction to align the tip to the load button of the weighting cell. However, a piezo stage moves the cantilever during the calibration procedure.

The $z$ position of the cantilever is measured with a second difference interferometer (SIOS SP $2000 \mathrm{DI})$. One of its beams is reflected on the beam balance while the other one hits the cantilever holder as shown in Figure 3.

This results in a short measurement loop (blue) that takes into account the thermal drift of the stages and the weighting cell.

\section{Results}

Figure 4 shows the force-deflection-curve of a cantilever that has been measured with the calibration device. Even with small calibration forces of less than $100 \mathrm{nN}$ the relative uncertainty of the cantilever stiffness is $1.5 \% \quad(k=2)$.

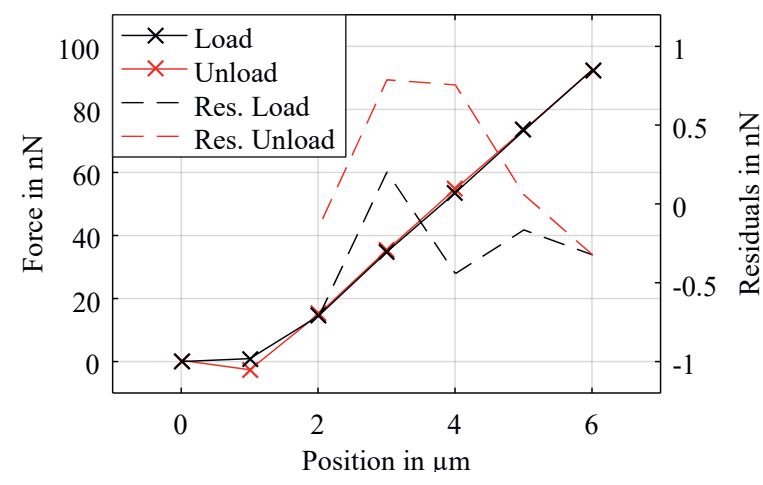

Fig. 4: Force-displacement-curve for one load cycle

After the calibration the cantilever tip has been examined under an electron microscope. The silicon tip with a $30 \mathrm{~nm}$ radius has not been damaged during the calibration process.

\section{References}

[1] C. A. Clifford et al., „The determination of atomic force microscope cantilever spring constants via dimensional methods for nanomechanical analysis“ Nanotechnology, 2005.

[2] C. Diethold, et al., „Determination of force to displacement curves using a nanopositioning system based on electromagnetic force compensated balances“ Measurement, 2014.

[3] C. Diethold, et al. „High precision optical position sensor for electromagnetic force compensated balances" in Proceedings of IMEKO, 2010. 